\title{
Spontaneous Raman spectra of dipicolinic acid in microcrystalline form
}

\author{
ALEXANDRE A. KOLOMENSKII, SERGEI N. JEREBTSOV, \\ TOMAS OPATRNY, HANS A. SCHUESSLER and \\ MARLAN O. SCULLY \\ Texas A\&M University, Department of Physics, College Station, \\ TX 77843-4242, USA
}

(Received 5 March 2003)

\begin{abstract}
Dipicolinic acid (DPA) is an important component of bacterial spores. The Raman spectrum of DPA in the form of compacted powder was measured in reflection at room temperature with excitation by a nanosecond laser at $532 \mathrm{~nm}$. The spectrum presents a set of characteristic frequency bands in the region $700-3090 \mathrm{~cm}^{-1}$ that were identified with characteristic vibrational modes of the DPA molecule.
\end{abstract}

\section{Introduction}

Dipicolinic acid (2,6-pyridinedicarboxylic acid, or briefly DPA) and its salts are major constituents of bacterial spores [1]. Recent interest in the development of robust spectroscopic methods of detection and identification of bacterial spores [2] is the motivation of the present study of the Raman spectra of solid DPA.

In this context, we note that previous resonance Raman spectra of Ghiamati et al. [3] involved DPA in aqueous solution. The present studies of the microcrystalline form are more readily compared with theory [4], because, for example, the resonance Raman data have in addition to the ground state vibrations also excited electronic state vibrations entering the physics of observed spectra. Furthermore, CaDPA in the spore corresponds more nearly to a solid sample than that of DPA in aqueous solution (see table 1).

Previous nonresonant Raman data of thin samples of DPA were obtained with transmission of light through thin films [5]. This data is also summarized in table 1. The Raman spectra were observed via excitation by a CW argon-ion laser at the wavelength of $514.5 \mathrm{~nm}$. However, the theoretical analysis of the vibrational frequencies of the DPA molecule [4] provided reasonable agreement with experimental data [5] only after the introduction of empirical correction factors. Thus, further experimental and theoretical studies of the DPA Raman spectra with crystalline and solution preparations are needed. In this paper, we present results of measurements of the DPA Raman spectra obtained from compacted powder samples. 
Table 1. Spectral peaks, vibrational modes and frequencies (in $\mathrm{cm}^{-1}$ ) of the DPA.

\begin{tabular}{llccc}
\hline $\begin{array}{l}\text { Raman at } \\
\text { 394 K (our } \\
\text { experiment) }\end{array}$ & Assignment & $\begin{array}{c}\text { Calculation [4] for } \\
\text { IR transitions }\end{array}$ & $\begin{array}{c}\text { Raman at } \\
\text { 100 K [5] }\end{array}$ & $\begin{array}{c}\text { Resonance Raman } \\
\text { spectroscopy* [3] }\end{array}$ \\
\hline 757 & $\begin{array}{c}\text { OCO in-plane } \\
\text { deformation }\end{array}$ & 741 & 762 & $\mathrm{np}$ \\
& $\begin{array}{c}\text { CH out-of-plane } \\
\text { deformation }\end{array}$ & 882 & 802 & $\mathrm{np}$ \\
& $\begin{array}{l}\text { C-H band } \\
892\end{array}$ & - & 895 & $\mathrm{np}$ \\
978 & $\begin{array}{l}\text { symmetric ring } \\
\text { "breathing" }\end{array}$ & 991 & 998 & 1000 \\
1063 & trigonal ring & $1077 * *$ & 1090 & 1070 \\
& "breathing" & C-H bend & & \\
1170 & C-H bend & $1123 * *$ & 1183 & 1196 \\
1253 & C-C ring mode & - & 1279 & 1272 \\
1416 & C-0 stretch & $1153 * *$ & 1304 & 1315 \\
1470 & C-C ring stretch & 1415 & 1447 & 1385 \\
1628 & C-C ring stretch & 1489 & 1475 & 1440 \\
1669 & C=C stretch & 1590 & 1640 & 1604 \\
2615 & C=O stretch & 1699 & 1646 & 1669 \\
3090 & O-H stretch & - & 2650 & $\mathrm{np}$ \\
& C-H stretch & 3088 & 3070 & $\mathrm{np}$ \\
\hline
\end{tabular}

$\mathrm{np}$ indicates that the spectral data were not presented for this wavelength region.

*Measurements were performed in aqueous solutions.

**The band nearest to the experimentally observed one is presented.

\section{Experimental arrangement}

The experimental setup is shown in figure 1. The sample was illuminated by the beam of the second harmonic of a Nd:YAG laser at $532 \mathrm{~nm}$. The scattered light was collected by a lens with a short focal length $(f=2.5 \mathrm{~cm})$ and then focused by a second lens with $f=10 \mathrm{~cm}$ into the entrance slit of a monochromator (McPherson, 1200 grooves/mm grating). The Rayleigh component of the scattered light was filtered out by two filters (Schott glass, OG550) of $2 \mathrm{~mm}$ thickness each. These filters were positioned immediately before the entrance slit of the monochromator and provided suppression of the spectral components at the excitation wavelength by a factor of more than $10^{8}$. The light from the exit slit of the monochromator was registered by a photomultiplier (EMI 9658R); the signal was amplified, averaged and recorded by a digital oscilloscope (TDS $680 \mathrm{C}, 1 \mathrm{GHz}$ real-time bandwidth). A portion of the excitation beam was deflected, registered by a photodiode and used to trigger the data acquisition process. Typically, the scan rate of the monochromator was $1 \mathrm{~nm} / \mathrm{min}$; the entrance slit was set to $300 \mu \mathrm{m}$, and the signal on the oscilloscope was averaged over 80 laser pulses. The averaged signal was transferred from the oscilloscope to a $\mathrm{PC}$ and the initial portion of this signal (about 75 data acquisition points with a temporal separation of $0.4 \mathrm{~ns}$ ) was integrated and recorded as a spectral response in each $10 \mathrm{~s}$ interval during the scan of the monochromator.

DPA in powder form was purchased from Sigma-Aldrich Corp. and used as such. For spectroscopic studies, the powder was pressed to the surface of a solid substrate (a plastic or fused silica plate) and formed a layer with about $1 \mathrm{~mm}$ thickness. The upper limit of the energy used for excitation $(2-4 \mathrm{~mJ})$ was 


\section{Experimental setup}

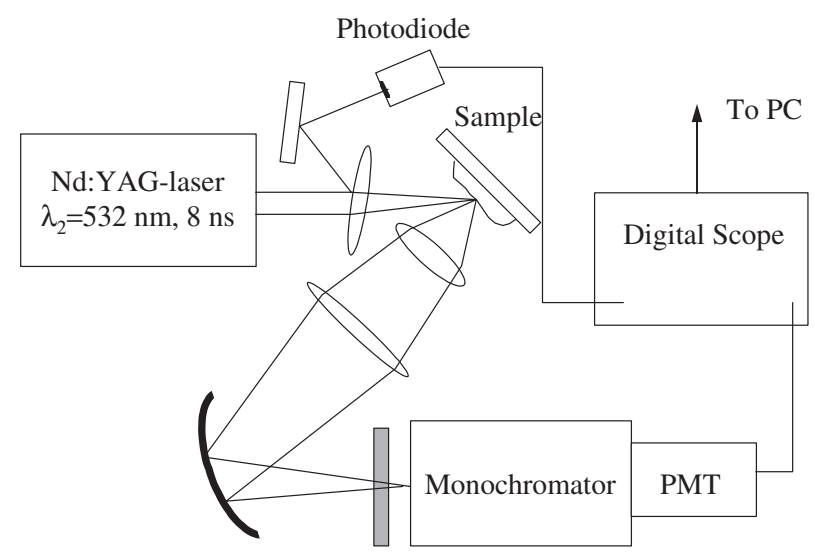

Figure 1. Schematic of the experimental setup. The excitation was performed by a nanosecond pulse of the second harmonic of a Nd:YAG laser at $532 \mathrm{~nm}$. The scattered light collected by the lenses was passed through the filter, the monochromator and then registered by the photomultiplier (PMT). The signal was amplified, averaged and recorded by a digital oscilloscope and transmitted to a $\mathrm{PC}$ computer for further processing.

determined by the laser fluence that DPA could stand without changes in its chemical composition. These changes could be noticed due to the appearance of a yellow shade on the initially white sample surface and due to changes in the observed spectral characteristics.

A spot of $5 \mathrm{~mm}$ in diameter was illuminated by the laser beam. Thus, the average fluence on the surface was below $0.01 \mathrm{~J} / \mathrm{cm}^{2}$ and the average intensity was below $10^{6} \mathrm{~J} /\left(\mathrm{s} \mathrm{cm}^{2}\right)$. In this case, only slight changes in the appearance and the spectral characteristics of the sample could be noticed after $30 \mathrm{~min}$ exposure to the laser radiation.

\section{Results of spectral measurements}

With powder preparations the sample surface was directly subjected to laser radiation. A typical Raman spectrum of a DPA powder preparation recorded at $1 \mathrm{~nm} / \mathrm{min}$ scan rate is shown in figure 2(a). This spectrum was obtained as the average of two scans and renormalized taking into account the transmission characteristic of the filters. It can be seen that the region of Raman shifts $700-1700 \mathrm{~cm}^{-1}$ contains many characteristic peaks that can be used for DPA identification. Some peaks were also observed below $700 \mathrm{~cm}^{-1}$, however strong absorption of the filters reduced the signal/noise ratio and did not allow precise measurement of the relatively small Raman shifts in this region. Attempts to use higher energy in laser pulses did not result in a better signal/noise ratio, on the contrary, it was noticed that with a higher laser fluence the Raman signal dropped significantly during the first several minutes of the measurement, obviously due to degradation of the sample. A Raman spectrum recorded with a somewhat lower resolution, but extending to higher frequencies, is shown in figure 2(b). At these higher frequencies only two additional Raman bands were registered. 
(a)

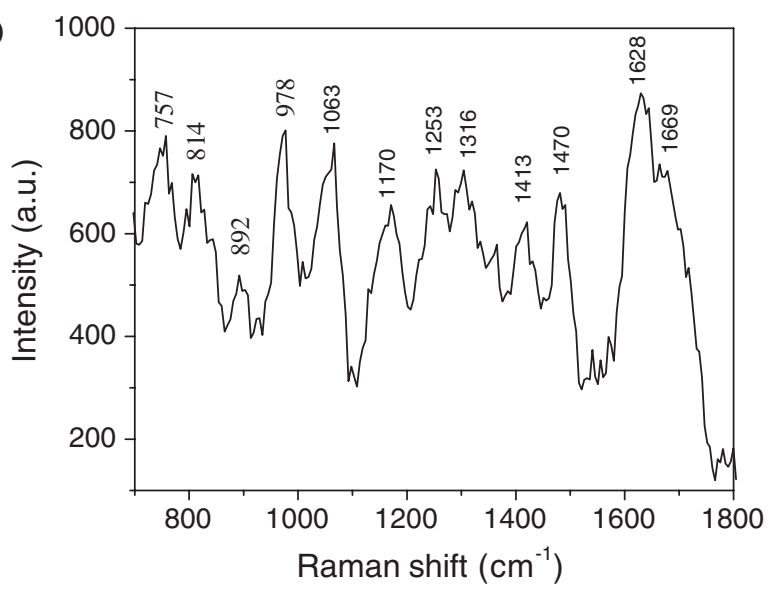

(b)

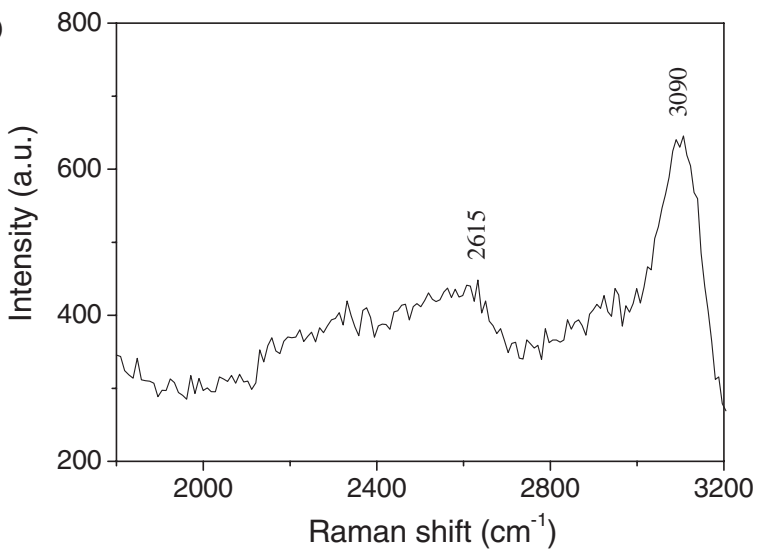

Figure 2. Raman spectra of DPA powder preparation: (a) in the region $700-1800 \mathrm{~cm}^{-1}$, (b) in the region $1800-3200 \mathrm{~cm}^{-1}$.

\section{Identification of the peaks in the Raman spectra}

The structure of the DPA molecule is shown in figure 3. The peaks observed in the Raman spectra correspond to characteristic vibrational modes of the DPA molecule (figure 4). For the same groups of atoms the frequency decreases in the sequence: stretching modes, planar (in-plane) bending vibrations, out-of-plane bending vibrations and torsion vibrations [7]. The vibrational frequency also increases with the strength of the bond (i.e. a double bond instead of a single bond) and with a decrease of the mass of the vibrating atom or atomic group. The measurements and interpretation of characteristic frequency regions allows rapid identification of the functional groups of the molecule and then identification of the molecule itself.

Identification of peaks in the Raman spectrum of DPA can be made based on the typical frequency ranges for certain vibrational modes [7.8] and on previously made assignments for DPA spectra $[4,5]$. Results are presented in table 1 . The ring 'breathing' band around $1000 \mathrm{~cm}^{-1}\left(978 \mathrm{~cm}^{-1}\right.$ in our measurements) is quite characteristic for the Raman spectra of benzene and pyridine [8] and was also 


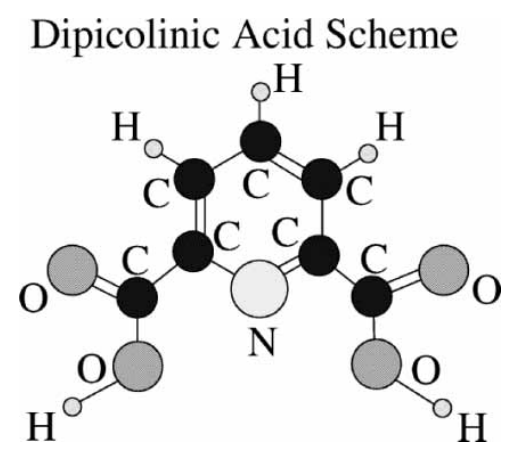

Figure 3. Structure of the dipicolinic acid molecule.
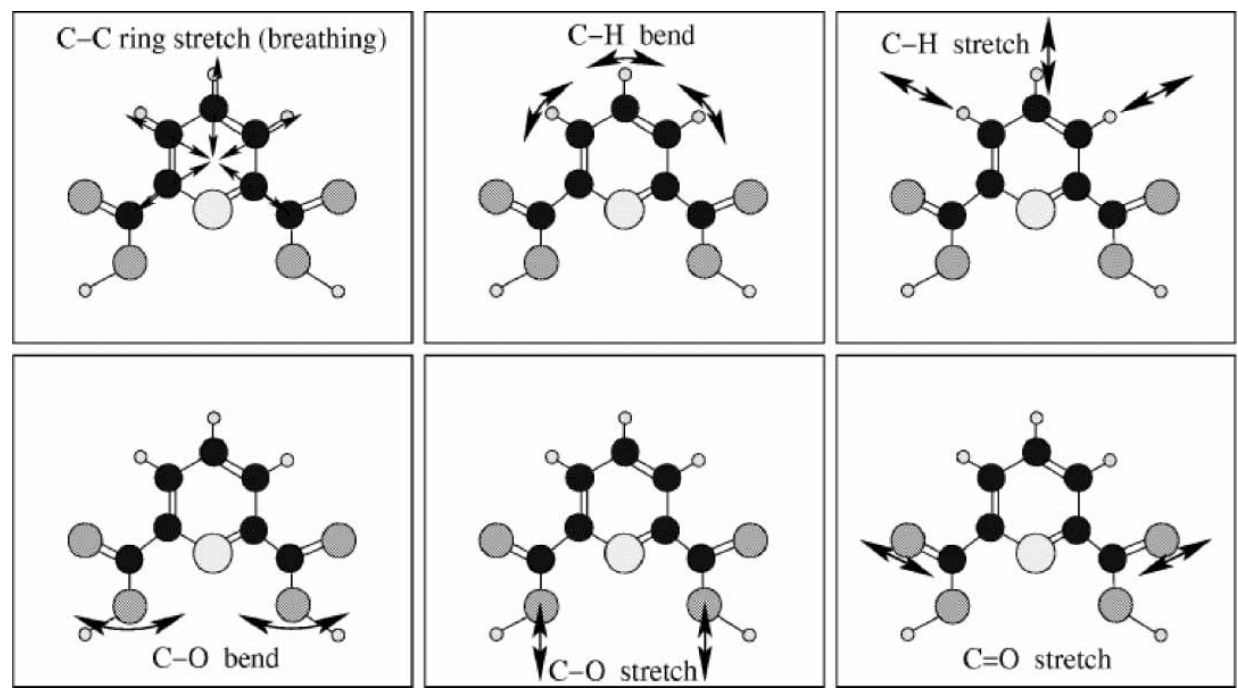

Figure 4. Illustration of prominent vibrational modes of the DPA molecule.

experimentally registered in DPA at $100 \mathrm{~K}$ [5]. The frequency of the trigonal ring 'breathing' band differs for various substituted pyridines, and we registered it at $1063 \mathrm{~cm}^{-1}$. The assignments of other peaks were made using data from earlier studies $[4,5,8]$.

\section{Discussion}

Comparison of the observed Raman spectra at room temperature with previously measured spectra at $100 \mathrm{~K}$ [5] shows close correspondence of the observed peaks above $1100 \mathrm{~cm}^{-1}$. The strong peaks in the bands of the 'breathing' modes appear differently, i.e. the symmetric ring 'breathing' band that we observed at $978 \mathrm{~cm}^{-1}$ in the measurements at $100 \mathrm{~K}$ [5] was assigned to the peak at $998 \mathrm{~cm}^{-1}$. Near $1063 \mathrm{~cm}^{-1}$, where we observed the trigonal ring 'breathing' band, at $100 \mathrm{~K}$ only a very weak peak of this band at $1090 \mathrm{~cm}^{-1}$ was detected [5].

In UV electronic resonance Raman spectra [3] measured at several wavelengths in the region $222-251 \mathrm{~nm}$, some bands are more pronounced at certain excitation 
wavelengths. For instance, the bands at $1060 \mathrm{~cm}^{-1}$ and $1272 \mathrm{~cm}^{-1}$ produce strong peaks in the scattered light for the excitation wavelength $231 \mathrm{~nm}$, while they are only slightly noticeable or absent at other excitation wavelengths. This reflects the general feature of UV resonance Raman spectra that relative intensities of spectral peaks may change erratically with small changes of excitation wavelength [9].

The OCO in-plane deformation mode at $757 \mathrm{~cm}^{-1}$, the $\mathrm{CH}$ out-of-plane bend mode at $817 \mathrm{~cm}^{-1}$, and the Raman scattering band at $892 \mathrm{~cm}^{-1}$ corresponding to $\mathrm{C}-\mathrm{H}$ band vibration were assigned following references [4, 5]. The Raman spectrum of pyridine [8] shows two bands at $990 \mathrm{~cm}^{-1}$ and $1030 \mathrm{~cm}^{-1}$ compared to one band at $1030 \mathrm{~cm}^{-1}$ in the region around $1000 \mathrm{~cm}^{-1}$ in benzene, which can be attributed to removal of the degeneracy of ring modes with substitution of $\mathrm{C}$ by $\mathrm{N}$. The presence of the carbonyl group can be identified by the $\mathrm{C}=\mathrm{O}$ stretch band around $1669 \mathrm{~cm}^{-1}$. Thus, the Raman spectrum of the DPA molecule exhibits a richness of spectral features useful for its identification.

\section{Conclusion}

Spontaneous Raman scattering spectra were measured for powder samples of $\mathrm{DPA}$ at room temperature with pulsed laser excitation at $532 \mathrm{~nm}$. These spectra were not affected by additional bands of the cuvette or solvent, as in the case of an aqueous solution, and exhibit many characteristic bands with frequencies mainly below $1700 \mathrm{~cm}^{-1}$. Above this frequency only $\mathrm{O}-\mathrm{H}$ and $\mathrm{C}-\mathrm{H}$ stretching modes were registered. Spectral bands below $700 \mathrm{~cm}^{-1}$ can also present useful spectroscopic information, however their detailed study is a matter for future work.

\section{Acknowledgements}

The authors appreciate stimulating discussions with M. S. Zubairy and A. V. Sokolov. This work was supported in part by Texas A\&M University, the US National Science Foundation and the Defense Advanced Research Projects Agency.

\section{References}

[1] Gould, G. W., and Hurst, A. (Eds.), 1969, The Bacterial Spore (London: Academic Press).

[2] Scully, M., Kattawar, G. W., Lucht, R. P., Opatrny, T., Pilloff, H., Rebane, A., Sokolov, A. V., and Zubairy, M. S., 2002, Proceedings of the National Academy of Sciences (PNAS), 99, 10994.

[3] Ghimati, E., Manoharan, R., Nelson, W. H., and Sperry, J. F., 1992, Appl. Spectrosc., 46, 357.

[4] Hameka, H. F., Jensen, J. O., Jensen, J. L., Merrow, C. N., and Vlahacos, C. P., 1996, J. Mol. Struct. (Theochem), 365, 131.

[5] Carmona, P., 1980, Spectrochim. Acta, 36A, 705.

[6] Kiefer, W., and Bernstein, H., 1971, Chem. Phys. Lett., 8, 381.

[7] Fadini, A., and Schnepel, F.-M., 1989, Vibrational Spectroscopy (New York: Wiley), pp. $40-62$.

[8] Dollish, F. R., Fateley, W. G., and Bentley, F. F., 1974, Characteristic Raman Frequencies of Organic Compounds (New York: Wiley), pp. 284-291.

[9] Rousseau, D. L., Friedman, J. M., and Williams, P. F., 1979, The resonance Raman effect. In Raman Spectroscopy of Gases and Liquids, edited by A. Weber (Heidelberg: Springer), pp. 203-252. 